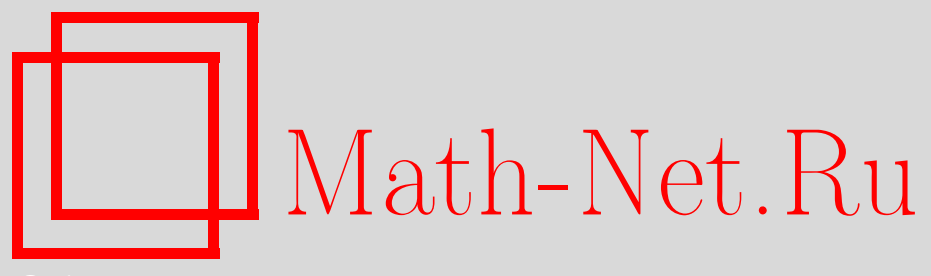

П. Н. Нестеров, Построение асимптотики решений одномерного уравнения Шрёдингера с быстро осциллирующим потенциалом, Матем. заметки, 2006, том 80, выпуск 2, 240-250

DOI: https://doi.org/10.4213/mzm2805

Использование Общероссийского математического портала Math-Net.Ru подразумевает, что вы прочитали и согласны с пользовательским соглашением http://www . mathnet.ru/rus/agreement

Параметры загрузки:

IP: 54.81 .137 .203

26 апреля 2023 г., $17: 30: 36$

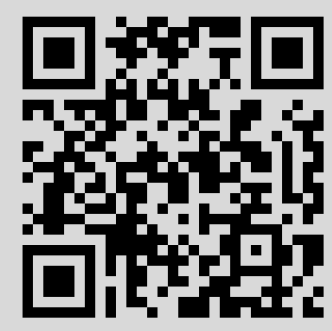




\section{ПОСТРОЕНИЕ АСИМПТОТИКИ РЕШЕНИЙ \\ ОДНОМЕРНОГО УРАВНЕНИЯ ШРЁДИНГЕРА \\ С БЫСТРО ОСЦИЛЛИРУЮЩИМ ПОТЕНЦИАЛОМ}

\section{П. Н. Нестеров}

Получены асимптотические формулы для решений одномерного уравнения Шрёдингера $-y^{\prime \prime}+q(x) y=0$ с осциллирующим потенциалом $q(x)=x^{\beta} P\left(x^{1+\alpha}\right)+$ $c x^{-2}$ при $x \rightarrow+\infty$. Действительные параметры $\alpha$ и $\beta$ удовлетворяют неравенствам $\beta-\alpha \geqslant-1,2 \alpha-\beta>0$, а $c$ - произвольная действительная постоянная. Действительная функция $P(x)$ является или периодической с периодом $T$, или тригонометрическим многочленом. При построении асимптотики применяются идеи метода усреднения, а также используется фундаментальная теорема Левинсона.

Библиография: 7 названий.

Рассмотрим одномерное уравнение Шрёдингера

$$
-y^{\prime \prime}+q(x) y=0
$$

на положительной полуоси $x>0$ с осциллирующим потенциалом $q(x)=x^{\beta} P\left(x^{1+\alpha}\right)+$ $c x^{-2}$. Здесь $c$ - произвольная действительная постоянная, а действительные параметры $\alpha$ и $\beta$ удовлетворяют следующим неравенствам:

$$
\beta-\alpha \geqslant-1, \quad 2 \alpha-\beta>0 .
$$

Эта задача исследовалась в работе [1] в предположении, что $P(x)$ - гладкая периодическая функция с нулевым средним значением. Мы будем предполагать, что $P(x)$ является или непрерывной периодической функцией с периодом $T$, или представляет собой тригонометрический многочлен вида

$$
P(x)=\sum_{j=0}^{n} p_{j} e^{i \lambda_{j} x}
$$

где $\lambda_{j}$ - произвольные действительные числа, а $p_{j}$ - комплексные числа. Мы также будем считать, что $P(x)$ является действительной функцией. В дальнейшем будем

Работа выполнена при финансовой поддержке ведомственной научной программы Министерства образования и науки РФ "Развитие научного потенциала высшей школы", проект № 49234, и программы “Университеты России”, грант № 04.01.452.

(C) П. Н. Нестеров, 2006 
говорить, что функция $P(x)$ принадлежит классу $\Sigma$, если она является периодической функцией или тригонометрическим многочленом. Кроме того, мы будем предполагать, что функция $P(x)$ имеет нулевое среднее значение, т.е.

$$
\lim _{T \rightarrow \infty} \frac{1}{T} \int_{0}^{T} P(x) d x=M(P(x))=0 .
$$

Здесь и везде далее $M(P(x))$ обозначает среднее периодической или почти периодической функции $P(x)$.

Сделаем в (1) замену независимой переменной $t=x^{1+\alpha}$. В терминах времени $t$ уравнение (1) примет вид

$$
\ddot{y}+\frac{\alpha}{1+\alpha} \frac{1}{t} \dot{y}-\frac{t^{(\beta-2 \alpha) /(1+\alpha)}}{(1+\alpha)^{2}} P(t) y-\frac{c}{(1+\alpha)^{2}} \frac{1}{t^{2}} y=0 .
$$

Для упрощения записи положим

$$
\gamma=\frac{\alpha}{1+\alpha}, \quad \delta=\frac{\beta-2 \alpha}{1+\alpha}, \quad \zeta=\frac{1}{(1+\alpha)^{2}} .
$$

Заметим, что из (2) и (4) непосредственно следует, что $-1 \leqslant \delta<0$. Далее нам предстоит проделать еще серию замен, но сперва перейдем от уравнения (3) стандартным образом к системе

$$
\dot{Y}_{0}=\left(\begin{array}{cc}
0 & 1 \\
\zeta t^{\delta} P(t) & -\gamma t^{-1}
\end{array}\right) Y_{0}+\left(\begin{array}{cc}
0 & 0 \\
c \zeta t^{-2} & 0
\end{array}\right) Y_{0}
$$

где

$$
Y_{0}=\left(\begin{array}{l}
y \\
\dot{y}
\end{array}\right)
$$

Пусть

$$
\phi(t)=-\int_{t}^{\infty} \zeta s^{\delta} P(s) d s .
$$

В силу сходимости интеграла эта функция определена и дифференцируема в каждой точке. Сделаем в (5) следующую замену:

$$
Y_{0}=\left(\begin{array}{cc}
1 & 0 \\
\phi(t) & 1
\end{array}\right) Y_{1}
$$

Тогда (5) трансформируется в

$$
\dot{Y}_{1}=\left(\begin{array}{cc}
\phi(t) & 1 \\
-\phi^{2}(t) & -\phi(t)-\gamma t^{-1}
\end{array}\right) Y_{1}+\left(\begin{array}{cc}
0 & 0 \\
c \zeta t^{-2}-\gamma \phi(t) t^{-1} & 0
\end{array}\right) Y_{1}
$$

Выберем функции $P_{1}(t)$ и $P_{2}(t)$ из класса $\Sigma$ с нулевым средним значением как решения следующих уравнений: $\dot{P}_{1}=P$ и $\dot{P}_{2}=P_{1}$. Проинтегрируем (6) два раза по частям, получим

$$
\phi(t)=-\left.\zeta s^{\delta} P_{1}(s)\right|_{t} ^{\infty}+\left.\zeta \delta s^{\delta-1} P_{2}(s)\right|_{t} ^{\infty}-\int_{t}^{\infty} \zeta\left(s^{\delta}\right)^{\prime \prime} P_{2}(s) d s .
$$


Откуда $\phi(t)=\zeta t^{\delta} P_{1}(t)+\phi_{1}(t)$, где $\phi_{1}(t)=-\zeta \delta t^{\delta-1} P_{2}(t)-\int_{t}^{\infty} \zeta\left(s^{\delta}\right)^{\prime \prime} P_{2}(s) d s$. Заметим, что $\phi_{1}(t)=O\left(t^{\delta-1}\right)$ при $t \rightarrow+\infty$. Далее,

$$
\phi^{2}(t)=\zeta^{2} t^{2 \delta} P_{1}^{2}(t)+2 \zeta t^{\delta} P_{1}(t) \phi_{1}(t)+\phi_{1}^{2}(t)=\zeta^{2} t^{2 \delta} P_{1}^{2}(t)-\zeta t^{\delta} \phi_{2}(t),
$$

где $\phi_{2}(t)=-2 P_{1}(t) \phi_{1}(t)-\left(\zeta t^{\delta}\right)^{-1} \phi_{1}^{2}(t)$. Несложно понять, что $\phi_{2}(t)=O\left(t^{\delta-1}\right), t \rightarrow$ $+\infty$. Теперь подставим полученные выражения для $\phi(t)$ и $\phi^{2}(t)$ в $(7)$ и осуществим так называемое срезающее преобразование, т.е. выполним замену

$$
Y_{1}=\left(\begin{array}{cc}
t^{-\delta / 2} & 0 \\
0 & t^{\delta / 2}
\end{array}\right) Y_{2}
$$

Тогда система (7) перейдет в систему следующего вида:

$$
\dot{Y}_{2}=\left(t^{\delta} A(t)+t^{-1} B(t)+t^{-2-\delta} C+R(t)\right) Y_{2} .
$$

Здесь

$$
\begin{aligned}
A(t) & =\left(\begin{array}{cc}
\zeta P_{1} & 1 \\
-\zeta^{2} P_{1}^{2} & -\zeta P_{1}
\end{array}\right), & B(t) & =\left(\begin{array}{cc}
\frac{\delta}{2} & 0 \\
-\gamma \zeta P_{1} & -\frac{\delta}{2}-\gamma
\end{array}\right), \\
C & =\left(\begin{array}{cc}
0 & 0 \\
\zeta c & 0
\end{array}\right), & R(t) & =\left(\begin{array}{cc}
\phi_{1}(t) & 0 \\
\zeta \phi_{2}(t)-\gamma \phi_{1}(t) t^{-1-\delta} & -\phi_{1}(t)
\end{array}\right) .
\end{aligned}
$$

Впрочем, конкретный вид матрицы $R(t)$ для нас в дальнейшем не имеет особого значения; главное, что о ней надо знать, это то, что

$$
R(t)=O\left(t^{\delta-1}\right), \quad t \rightarrow+\infty .
$$

Следовательно, элементы матрицы $R(t)$ абсолютно интегрируемы на $\left[t_{0}, \infty\right)$. Мы будем писать $R(t) \in L_{1}\left[t_{0}, \infty\right)$, понимая под этим, что $\|R(t)\| \in L_{1}\left[t_{0}, \infty\right)$, где $\|\cdot\|-$ некоторая индуцированная матричная норма. Заметим также, что элементы матриц $A(t)$ и $B(t)$ принадлежат классу $\Sigma$. Для дальнейшего анализа системы (8) мы будем использовать известный метод усреднения (см. [2]). Его применение к построению асимптотики линейных дифференциальных уравнений с колебательно убывающими коэффициентами было разработано в [3]. Кратко изложим суть метода.

Рассмотрим систему

$$
\frac{d \hat{x}}{d t}=\left\{A_{0}+\sum_{j=1}^{k} \frac{1}{t^{j \alpha}} A_{j}(t)\right\} \hat{x}+O\left(t^{-\varphi_{1}}\right) \hat{x} .
$$

Здесь $A_{0}$ - постоянная квадратная матрица порядка $n$, все собственные значения которой вещественны, а элементами матриц $A_{1}(t), A_{2}(t), \ldots, A_{k}(t)$ являются тригонометрические многочлены. Вещественное число $\alpha$ и натуральное число $k$ удовлетворяют неравенствам $0<k \alpha \leqslant 1<(k+1) \alpha, \varphi_{1}>1$. Система уравнений (9) при достаточно больших $t$ с помощью замены

$$
\hat{x}=\left\{\sum_{j=0}^{k} \frac{1}{t^{j \alpha}} V_{j}(t)\right\} \hat{y},
$$


где $V_{0}(t)=I$ - единичная матрица, а элементами матриц $V_{j}(t), j=1, \ldots, k$, являются тригонометрические многочлены с нулевым средним значением, преобразуется в систему

$$
\frac{d \hat{y}}{d t}=\left\{A_{0}+\sum_{j=1}^{k} \frac{1}{t^{j \alpha}} A_{j}\right\} \hat{y}+O\left(t^{-\varphi_{2}}\right) \hat{y}
$$

где $\left\{A_{j}\right\}$ - постоянные матрицы. Константа $\varphi_{2}$ выбирается следующим образом: $\varphi_{2}=\min \left\{(1+k) \alpha, \varphi_{1}\right\}$. Матрицы $V_{j}(t)$ с нулевым средним значением определяются как решения следующих неоднородных дифференциальных уравнений с постоянными коэффициентами:

$$
\frac{d V_{j}(t)}{d t}-A_{0} V_{j}(t)+V_{j}(t) A_{0}=\sum_{l=0}^{j-1} A_{j-l}(t) V_{l}(t)-\sum_{l=0}^{j-1} V_{l}(t) A_{j-l} .
$$

На каждом шаге $j=1, \ldots, k$ матрица $A_{j}$ определяется из условия, что правая часть системы (12) имеет нулевое среднее значение

$$
A_{j}=M\left(\sum_{l=0}^{j-1} A_{j-l}(t) V_{l}(t)\right)
$$

В частности, $A_{1}=M\left(A_{1}(t)\right), A_{2}=M\left(A_{2}(t)+A_{1}(t) V_{1}(t)\right)$.

$\mathrm{B}$ нашем случае исходная система несколько отличается от системы (9). Более точно, она имеет следующий вид:

$$
\frac{d \hat{x}}{d t}=\left\{\sum_{j=1}^{k} \frac{1}{t^{j \alpha}} A_{j}(t)\right\} \hat{x}+\xi(t) B(t) \hat{x}+O\left(t^{-\varphi_{1}}\right) \hat{x} .
$$

Здесь матрицы $A_{1}(t), A_{2}(t), \ldots, A_{k}(t)$ суть матрицы с элементами из класса $\Sigma$. Вещественное число $\alpha$ и натуральное число $k$ удовлетворяют неравенствам $0<k \alpha \leqslant$ $1<(k+1) \alpha, \varphi_{1}>1$. Матрица $B(t)$ ограничена, т.е. $\|B(t)\| \leqslant C<\infty$ при $t \geqslant t_{0}$, функция $\xi(t)=O\left(t^{-\beta}\right)$ и $\alpha+\beta>1$. В этом случае система уравнений (14) при достаточно больших $t$ с помощью замены (10) преобразуется в систему

$$
\frac{d \hat{y}}{d t}=\left\{\sum_{j=1}^{k} \frac{1}{t^{j \alpha}} A_{j}\right\} \hat{y}+\xi(t) B(t) \hat{y}+O\left(t^{-\varphi_{2}}\right) \hat{y}
$$

где матрицы $V_{j}(t)$ и $A_{j}, j=1, \ldots, k$, определяются из $(12)$ и (13) соответственно. Значение константы $\varphi_{2}$ определяется следующим образом: $\varphi_{2}=\min \{(1+k) \alpha, \alpha+$ $\left.\beta, \varphi_{1}\right\}$. Осталось заметить, что если $A_{0}=0$, то система (12) однозначно разрешима в классе периодических матриц с нулевым средним значением, если элементами матриц $A_{j}(t), j=1, \ldots, k$, являются непрерывные периодические функции с периодом $T>0$. В этом случае элементами матриц $V_{j}(t), j=1, \ldots, k$, являются $T$-периодические функции с нулевым средним значением.

На следующем этапе для получения асимптотики используется теорема Левинсона (см., например, [4], [5]). Приведем ее формулировку применительно к системе (11). 
Теорема. Пусть среди матрии $A_{j}, j=0, \ldots, k$, первой ненулевой является матрица $A_{l}$. Пусть матрица $A_{l}$ имеет различные собственные значения. Тогда фундаментальная матрица системы (11) имеет вид

$$
\Phi(t)=(\Pi+o(1)) \exp \left\{\int_{t^{*}}^{t} \Lambda(s) d s\right\}, \quad t>t^{*}, \quad t \rightarrow \infty,
$$

где П - матрица, столбцы которой состоят из собственных векторов матрицљь $A_{l}, u \Lambda(t)$ - диагональная матрица, элементами которой являются собственные значения матрицы $\sum_{j=l}^{k} t^{-j \alpha} A_{j}$.

Замена

$$
Y_{2}=\left(I+\frac{1}{t} V(t)\right) Y_{3}
$$

где $V(t)$ - матрица с нулевым средним значением и с элементами из класса $\Sigma$, приводит систему (8) к виду

$$
\dot{Y}_{3}=\left(t^{\delta} A(t)+t^{-1} B+t^{-2-\delta} C+O\left(t^{\delta-1}\right)\right) Y_{3},
$$

где

$$
B=M(B(t))=\left(\begin{array}{cc}
\frac{\delta}{2} & 0 \\
0 & -\frac{\delta}{2}-\gamma
\end{array}\right) .
$$

Поскольку при различных $\delta$ асимптотика решений будет существенным образом отличаться, нам следует рассмотреть несколько случаев.

Случай I. $\delta=-1$ (или, что эквивалентно, $\beta-\alpha=-1)$. Заменой

$$
Y_{3}=\left(I+\frac{1}{t} V(t)\right) Y
$$

мы перейдем от (15) к системе

$$
\dot{Y}=\left(\frac{1}{t}\left(A_{1}+B+C\right)+O\left(t^{-2}\right)\right) Y,
$$

где

$$
A_{1}=M(A(t))=\left(\begin{array}{cc}
0 & 1 \\
-\zeta^{2} a & 0
\end{array}\right)
$$

Здесь и далее

$$
a=M\left(P_{1}^{2}(t)\right)=M\left[\left(\int_{0}^{t} P(s) d s\right)^{2}\right]-\left[M\left(\int_{0}^{t} P(s) d s\right)\right]^{2} \geqslant 0 .
$$

Вычислим собственные числа матрицы

$$
A_{1}+B+C=\left(\begin{array}{cc}
-\frac{1}{2} & 1 \\
-\zeta^{2} a+\zeta c & \frac{1}{2}-\gamma
\end{array}\right)
$$


В зависимости от знака дискриминанта характеристического многочлена рассмотрим два варианта.

1. Пусть $a>(c+1 / 4)(1+\alpha)^{2}$. Тогда

$$
\lambda_{1,2}=-\frac{\gamma}{2} \pm \zeta i \sqrt{a-\left(c+\frac{1}{4}\right)(1+\alpha)^{2}} .
$$

Матрица П имеет вид

$$
\left(\begin{array}{cc}
1 & 1 \\
\pi_{21} & \pi_{22}
\end{array}\right)
$$

Легко видеть, что

$$
\exp \left\{\int_{t^{*}}^{t} \Lambda(s) d s\right\}=\left(\begin{array}{cc}
\exp \left\{\lambda_{1} \ln \frac{t}{t^{*}}\right\} & 0 \\
0 & \exp \left\{\lambda_{2} \ln \frac{t}{t^{*}}\right\}
\end{array}\right)
$$

Используя теорему 1 для построения асимптотики фундаментальной матрицы системы (16) и вспоминая все проделанные нами замены, получаем асимптотику для линейно независимых решений $y_{1,2}(x)$ уравнения (1) при $x \rightarrow+\infty$ :

$$
y_{1,2}(x)=x^{1 / 2} \exp \left\{ \pm i \omega(1+\alpha)^{-1} \ln x\right\}(1+o(1)),
$$

где $\omega=\sqrt{a-(c+1 / 4)(1+\alpha)^{2}}$.

2. Пусть теперь $a<(c+1 / 4)(1+\alpha)^{2}$. Тогда

$$
\lambda_{1,2}=-\frac{\gamma}{2} \pm \zeta \sqrt{-\left[a-\left(c+\frac{1}{4}\right)(1+\alpha)^{2}\right]}
$$

и легко видеть, что

$$
\exp \left\{\int_{t^{*}}^{t} \Lambda(s) d s\right\}=\left(\begin{array}{cc}
{\frac{t}{t^{*}}}^{\lambda_{1}} & 0 \\
0 & \frac{t^{\lambda_{2}}}{t^{*}}
\end{array}\right) .
$$

Несложно установить, что в этом случае решения $y_{1,2}(x)$ имеют при $x \rightarrow+\infty$ следующую асимптотику:

$$
y_{1,2}(x)=x^{(1 / 2) \pm \omega_{*}(1+\alpha)^{-1}}(1+o(1))
$$

где $\omega_{*}=\sqrt{-\left[a-(c+1 / 4)(1+\alpha)^{2}\right]}$.

Случай II. $-1<\delta<-1 / 2$. Заменой $Y_{3}=\left(I+t^{\delta} V(t)\right) Y$ мы преобразуем (15) в систему

$$
\dot{Y}=\left(t^{\delta} A_{1}+t^{-1} B+O\left(t^{-\varphi_{2}}\right)\right) Y
$$

где $\varphi_{2}=\min \{-2 \delta, 1-\delta, \delta+2\}$. Вычислим собственные числа матрицы

$$
t^{\delta} A_{1}+t^{-1} B=\left(\begin{array}{cc}
\frac{\delta}{2} t^{-1} & t^{\delta} \\
-\mu t^{\delta} & -\frac{\delta}{2} t^{-1}-\gamma t^{-1}
\end{array}\right)
$$


где для сокращения записи принято обозначение $\mu=\zeta^{2} a>0$. Обозначим также величину $\zeta \sqrt{a}$ через $\nu$. Собственные значения имеют вид

$$
\lambda_{1,2}(t)=-\frac{\gamma}{2} t^{-1} \pm i \nu t^{\delta} \sqrt{1-\frac{(\gamma+\delta)^{2}}{4 \mu} t^{-2(1+\delta)}}=-\frac{\gamma}{2} t^{-1} \pm i\left(\nu t^{\delta}+O\left(t^{-2-\delta}\right)\right)
$$

когда $t \rightarrow+\infty$. Здесь мы воспользовались тейлоровским разложением для $\sqrt{1+x}$ при $x \rightarrow 0$. Матрица П имеет теперь вид

$$
\left(\begin{array}{cc}
1 & 1 \\
i \nu & -i \nu
\end{array}\right)
$$

$\mathrm{a} \exp \left\{\int_{t^{*}}^{t} \Lambda(s) d s\right\}$ выглядит следующим образом:

$$
\left(\begin{array}{cc}
t^{-\gamma / 2} \exp \left\{i\left(\nu \frac{t^{\delta+1}}{\delta+1}+O\left(t^{-\delta-1}\right)\right)\right\} & 0 \\
0 & t^{-\gamma / 2} \exp \left\{-i\left(\nu \frac{t^{\delta+1}}{\delta+1}+O\left(t^{-\delta-1}\right)\right)\right\}
\end{array}\right) \Delta .
$$

Здесь через $\Delta$ обозначена постоянная диагональная матрица, зависящая от $t^{*}$. Возвращаясь к исходной независимой переменной $x$, имеем

$$
y_{1,2}(x)=x^{(\alpha-\beta) / 2} \exp \{ \pm i S(x)\}(1+o(1))
$$

где $S(x)=\sqrt{a}(1+\alpha)^{-1}(\beta-\alpha+1)^{-1} x^{\beta-\alpha+1}$.

Случай III. $-1 / 2 \leqslant \delta<-1 / 3$. С помощью замены $Y_{3}=\left(I+t^{\delta} V_{1}(t)+t^{2 \delta} V_{2}(t)\right) Y$ от системы (15) мы переходим к системе

$$
\dot{Y}=\left(t^{\delta} A_{1}+t^{2 \delta} A_{2}+t^{-1} B+O\left(t^{-\varphi_{2}}\right)\right) Y .
$$

Здесь

$$
A_{2}=M\left(A_{1}(t) V_{1}(t)\right)=\left(\begin{array}{cc}
0 & 0 \\
-\varphi & 0
\end{array}\right)
$$

где $\varphi=2 \zeta^{3} M\left[\int_{0}^{t} P_{1}(s) d s\left(P_{1}^{2}(t)-a\right)\right]$ и $\varphi_{2}=\min \{-3 \delta, 1-\delta, 1-\delta\}=-3 \delta$. Вычисляя собственные числа матрицы

$$
t^{\delta} A_{1}+t^{2 \delta} A_{2}+t^{-1} B=\left(\begin{array}{cc}
\frac{\delta}{2} t^{-1} & t^{\delta} \\
-\mu t^{\delta}-\varphi t^{2 \delta} & -\frac{\delta}{2} t^{-1}-\gamma t^{-1}
\end{array}\right)
$$

имеем

$$
\lambda_{1,2}(t)=-\frac{\gamma}{2} t^{-1} \pm i \nu t^{\delta} \sqrt{1-\frac{(\gamma+\delta)^{2}}{4 \mu} t^{-2(1+\delta)}+\frac{\varphi}{\mu} t^{\delta}} .
$$

Как и в предыдущем случае, разложим $\lambda_{1,2}(t)$ по формуле Тейлора. Тогда

$$
\lambda_{1,2}(t)=-\frac{\gamma}{2} t^{-1} \pm i\left(\nu t^{\delta}+\frac{\varphi \nu}{2 \mu} t^{2 \delta}+O\left(t^{3 \delta}\right)\right), \quad t \rightarrow+\infty,
$$


где было учтено, что $1<-3 \delta \leqslant \delta+2$. Далее, действуя так же, как и ранее, приходим к выводу, что

$$
y_{1,2}(x)=x^{(\alpha-\beta) / 2} \exp \{ \pm i S(x)\}(1+o(1)), \quad x \rightarrow+\infty .
$$

Функция $S(x)$ выглядит следующим образом:

$$
S(x)=\sqrt{a}(1+\alpha)^{-1}(\beta-\alpha+1)^{-1} x^{\beta-\alpha+1}+\frac{\widehat{M} \ln x}{\sqrt{a}(1+\alpha)^{3}}, \quad \delta=-\frac{1}{2},
$$

и

$$
S(x)=\sqrt{a}(1+\alpha)^{-1}(\beta-\alpha+1)^{-1} x^{\beta-\alpha+1}+\frac{\widehat{M}}{\sqrt{a}(1+\alpha)^{3}} \frac{x^{-3 \alpha+2 \beta+1}}{(-3 \alpha+2 \beta+1)}, \quad \delta>-\frac{1}{2},
$$

где $\widehat{M}=M\left[\int_{0}^{t} P_{1}(s), d s\left(P_{1}^{2}(t)-a\right)\right]$.

Случай IV. $-1 / k \leqslant \delta<-1 /(k+1), k>2, k$ целое. Для исследования этого случая нам потребуется доказать одно вспомогательное утверждение. Рассмотрим систему дифференциальных уравнений

$$
\frac{d \hat{x}}{d t}=A(t) \hat{x}+O\left(\varepsilon^{k+1}\right) \hat{x}
$$

где $A(t)=\sum_{l=1}^{k} \varepsilon^{l} A_{l}(t), \varepsilon>0$ - малый параметр, $A_{l}(t)$ - матрицы с элементами из класса $\Sigma$. Как известно (см. [6]), система (17) заменой

$$
\hat{x}=\left(I+\sum_{l=1}^{k} \varepsilon^{l} V_{l}(t)\right) \hat{y}
$$

где $V_{l}(t), l=1, \ldots, k,-$ матрицы с элементами из класса $\Sigma$ с нулевым средним, приводится к виду

$$
\frac{d \hat{y}}{d t}=\left(\sum_{l=1}^{k} \varepsilon^{l} A_{l}\right) \hat{y}+O\left(\varepsilon^{k+1}\right) \hat{y}
$$

где $A_{l}$ - постоянные матрицы.

УтВЕРЖДЕНИЕ 1. Пусть $M\left(\operatorname{tr} A_{l}(t)\right)=0, l=1, \ldots, k$. Тогда все матрицъ $A_{l}$ имеют нулевой след.

ДокАЗАтЕльство 1. Выполняя замену (18), получим равенство

$$
\left(I+\sum_{l=1}^{k} \varepsilon^{l} V_{l}(t)\right) \frac{d \hat{y}}{d t}+\left(\sum_{l=1}^{k} \varepsilon^{l} \frac{d V_{l}}{d t}\right) \hat{y}=A(t)\left(I+\sum_{l=1}^{k} \varepsilon^{l} V_{l}(t)\right) \hat{y}+O\left(\varepsilon^{k+1}\right) \hat{y} .
$$

Отсюда

$$
\begin{aligned}
\frac{d \hat{y}}{d t}+ & \left(I+\sum_{l=1}^{k} \varepsilon^{l} V_{l}(t)\right)^{-1}\left(\sum_{l=1}^{k} \varepsilon^{l} \frac{d V_{l}}{d t}\right) \hat{y} \\
& =\left(I+\sum_{l=1}^{k} \varepsilon^{l} V_{l}(t)\right)^{-1} A(t)\left(I+\sum_{l=1}^{k} \varepsilon^{l} V_{l}(t)\right) \hat{y}+O\left(\varepsilon^{k+1}\right) \hat{y}
\end{aligned}
$$


Учитывая (19), получаем матричное равенство

$$
\begin{gathered}
\left(\sum_{l=1}^{k} \varepsilon^{l} A_{l}\right)+O\left(\varepsilon^{k+1}\right)+\left(I+\sum_{l=1}^{k} \varepsilon^{l} V_{l}(t)\right)^{-1}\left(\sum_{l=1}^{k} \varepsilon^{l} \frac{d V_{l}}{d t}\right) \\
=\left(I+\sum_{l=1}^{k} \varepsilon^{l} V_{l}(t)\right)^{-1} A(t)\left(I+\sum_{l=1}^{k} \varepsilon^{l} V_{l}(t)\right) .
\end{gathered}
$$

Вычислим след левой и правой части. Так как подобные матрицы имеют одинаковый след, то след правой части равен $\operatorname{tr} A(t)$. Имеем

$$
\operatorname{tr}\left[\left(I+\sum_{l=1}^{k} \varepsilon^{l} V_{l}(t)\right)^{-1}\left(\sum_{l=1}^{k} \varepsilon^{l} \frac{d V_{l}}{d t}\right)\right]=-\left(\sum_{l=1}^{k} \varepsilon^{l} \operatorname{tr} A_{l}\right)+\operatorname{tr} A(t)+O\left(\varepsilon^{k+1}\right) .
$$

Разложим левую часть (20) в ряд по степеням $\varepsilon$, предварительно обозначив $F(t)=$ $-\sum_{l=1}^{k} \varepsilon^{l} V_{l}(t)$. При достаточно малых $\varepsilon$ матрица $I-F$ обратима и $(I-F)^{-1}=$ $\sum_{n=0}^{\infty} F^{n}$. Левая часть (20) тогда запишется в виде

$$
-\operatorname{tr}\left(\sum_{n=0}^{\infty} F^{n} \dot{F}\right)=-\sum_{n=0}^{\infty} \operatorname{tr}\left(F^{n} \dot{F}\right)=-\sum_{n=1}^{\infty} \varepsilon^{n} f_{n}(t)
$$

где $f_{j}(t)$ - функции из класса $\Sigma$, полученные в результате объединения слагаемых в $\sum_{n=0}^{\infty} \operatorname{tr}\left(F^{n} \dot{F}\right)$ при одинаковых степенях $\varepsilon$. Для дальнейшего доказательства нам потребуется одно несложное соображение.

Если $F(t)$ - дифференцируемая матрица, то

$$
\operatorname{tr}\left(F^{k+1}(t)\right)^{\prime}=\left(\operatorname{tr} F^{k+1}(t)\right)^{\prime}=(k+1) \operatorname{tr}\left(F^{k}(t) F^{\prime}(t)\right) .
$$

Это следует из равенства

$$
\left(F^{k+1}(t)^{\prime}\right)=F^{\prime}(t) F^{k}(t)+F(t) F^{\prime}(t) F^{k-1}(t)+F^{2}(t) F^{\prime}(t) F^{k-2}(t)+\cdots+F^{k}(t) F^{\prime}(t)
$$

и того факта, что $\operatorname{tr} A B=\operatorname{tr} B A$. Из (22) следует, что

$$
M\left[\operatorname{tr}\left(F^{k}(t) F^{\prime}(t)\right)\right]=0 .
$$

Вычислим средние значения левой и правой частей равенства (21). Среднее значение левой части равно нулю ввиду равенства (23). Таким образом,

$$
\sum_{n=1}^{\infty} \varepsilon^{n} M\left(f_{n}(t)\right)=0
$$

Откуда сразу же вытекает, что $M\left(f_{j}(t)\right)=0$ для всех $j$. Приравняем слагаемые в левой и правой части (20) при одинаковых степенях $\varepsilon$ и возьмем среднее значение от обеих частей в полученном равенстве. Учтем, что $M\left(f_{j}(t)\right)=0$ для всех $j$, а также то, что $M\left(\operatorname{tr} A_{l}(t)\right)=0, \quad l=1, \ldots, k$. Получим, что $\operatorname{tr} A_{l}=0, \quad l=1, \ldots, k$. Утверждение доказано. 
Пусть теперь в (17)-(19) $\varepsilon=\varepsilon(t), \varepsilon(t) \rightarrow 0$ при $t \rightarrow \infty$ и вместо $O\left(\varepsilon^{k+1}\right)$ стоят некоторые матрицы из класса $L_{1}\left[t_{0}, \infty\right)$. Пусть также $\dot{\varepsilon}(t) \in L_{1}\left[t_{0}, \infty\right), \varepsilon(t), \ldots, \varepsilon^{k}(t) \notin$ $L_{1}\left[t_{0}, \infty\right)$, а $\varepsilon^{k+1}(t) \in L_{1}\left[t_{0}, \infty\right)$. Если выполнены остальные условия утверждения 1 , то $\operatorname{tr} A_{l}=0, l=1, \ldots, k$.

Доказательство этого утверждения почти дословно совпадает с доказательством утверждения 1. Утверждение остается справедливым и в случае, когда рассматриваемая система имеет вид

$$
\frac{d \hat{x}}{d t}=A(t) \hat{x}+\xi(t) B(t) \hat{x}+G(t) \hat{x}
$$

где относительно $A(t)$ приняты те же предположения, что и выше, $G(t) \in L_{1}\left[t_{0}, \infty\right)$, $B(t)$ ограничена, а $\xi(t) \varepsilon(t) \in L_{1}\left[t_{0}, \infty\right)$.

Итак, заменой $Y_{3}=\left(I+t^{\delta} V_{1}(t)+\cdots+t^{k \delta} V_{k}(t)\right) Y$ от системы (15) мы переходим к системе

$$
\dot{Y}=\left(t^{\delta} A_{1}+\cdots+t^{k \delta} A_{k}+t^{-1} B+O\left(t^{-\varphi_{2}}\right)\right) Y,
$$

где $\varphi_{2}=\min \{-(1+k) \delta, 1-\delta, 1-\delta\}=-(1+k) \delta$. В этом случае нам необходимо определить собственные числа матрицы

$$
t^{\delta} A_{1}+t^{2 \delta} A_{2}+\cdots+t^{k \delta} A_{k}+t^{-1} B=\left(\begin{array}{cc}
\frac{\delta}{2} t^{-1}+\Psi(t) & t^{\delta}+\cdots \\
-\mu t^{\delta}-\varphi t^{2 \delta}+\cdots & -\frac{\delta}{2} t^{-1}-\gamma t^{-1}-\Psi(t)
\end{array}\right)
$$

где $\Psi(t)=\psi_{l} t^{l \delta}+\cdots, \quad 3 \leqslant l \leqslant k$, а под многоточием подразумеваются члены вида $c_{m} t^{m \delta}$ более высокого порядка малости. Имеем

$$
\lambda_{1,2}(t)=-\frac{\gamma}{2} t^{-1} \pm i \nu t^{\delta} \sqrt{1-\frac{(\gamma+\delta)^{2}}{4 \mu} t^{-2(1+\delta)}+\frac{\varphi}{\mu} t^{\delta}+u_{l} t^{(l-2) \delta-1}+\cdots}
$$

где на сей раз под многоточием следует понимать слагаемые вида $r_{m} t^{m \delta}, m \geqslant 2$, и $u_{r} t^{(r-2) \delta-1}, r>l$. Далее, раскладывая собственные числа по формуле Тейлора, имеем

$$
\lambda_{1,2}(t)=-\frac{\gamma}{2} t^{-1} \pm i\left(\nu t^{\delta}+\frac{\varphi \nu}{2 \mu} t^{2 \delta}+O\left(t^{3 \delta}\right)+O\left(t^{(k+1) \delta}\right)\right), \quad t \rightarrow+\infty .
$$

В этом выражении мы учли, что главным слагаемым среди тех, которые принадлежат $L_{1}\left[t_{0}, \infty\right)$, в асимптотическом разложении для $\lambda_{1,2}(t)$ является именно $O\left(t^{(k+1) \delta}\right)$. Вычисляя $\exp \left\{\int_{t^{*}}^{t} \Lambda(s) d s\right\}$, получаем асимптотику для линейно независимых решений $y_{1,2}(x)$ уравнения $(1)$

$$
y_{1,2}(x)=x^{(\alpha-\beta) / 2} \exp \{ \pm i S(x)\}(1+o(1)), \quad x \rightarrow+\infty .
$$

Функция $S(x)$ имеет вид

$$
\begin{aligned}
S(x)=\sqrt{a} & (1+\alpha)^{-1}(\beta-\alpha+1)^{-1} x^{\beta-\alpha+1} \\
& +\frac{\widehat{M}}{\sqrt{a}(1+\alpha)^{3}} \frac{x^{-3 \alpha+2 \beta+1}}{(-3 \alpha+2 \beta+1)}+O\left(x^{-5 \alpha+3 \beta+1}\right) .
\end{aligned}
$$


Если $-5 \alpha+3 \beta+1=0$ (т.е. $\delta=-1 / 3)$, то вместо $O\left(x^{-5 \alpha+3 \beta+1}\right)$ следует писать $O(\ln x)$.

Полученные асимптотические формулы остаются справедливыми и при менее ограничительных предположениях относительно функции $P(x)$. Достаточно считать, что периодическая функция $P(x)$ интегрируема по Риману на промежутке $[0, T]$.

В заключение отметим также, что можно оценить скорость стремления к нулю члена $о(1)$ в асимптотических формулах. Соответствующие результаты приведены в работе [7].

Автор благодарит профессора В.Ш. Бурда за постановку задачи и внимание к работе.

\section{СПИСОК ЦИТИРОВАННОЙ ЛИТЕРАТУРЫ}

[1] А. Р. Итс, “Асимптотическое поведение решений радиального уравнения Шрёдингера с осциллирующим потенциалом при нулевой энергии”, Проблемы математической физики, Сб. статей, № 9, Изд-во Ленинградского ун-та, Ленинград, 1979, 30-41.

[2] Н.Н.Боголюбов, Ю.А. Митропольский, Асимптотические методы в теории нелинейных колебаний, Наука, М., 1974.

[3] В.Ш. Бурд, В.А. Каракулин, "Асимптотическое интегрирование систем линейных дифференциальных уравнений с колебательно убывающими коэффициентами", Матем. заметки, 64:5 (1998), 658-666.

[4] Б.П. Демидович, Лекиии по математической теории устойчивости, Наука, М., 1967.

[5] Э. А. Коддингтон, Н. Левинсон, Теория обыкновенных дифференциалъных уравнений, ИЛ, М., 1958.

[6] И. З. Штокало, Линейные дифференциальные уравнения с переменными коэффициентами, Изд-во АН УССР, Киев, 1960.

[7] П. Н. Нестеров, Асимптотическое поведение решений одномерного уравнения Шрёдингера с быстро осциллирующим потенциалом, Рук. деп. в ВИНИТИ РАН 29.04.2005, № 640-B, 2005.

\section{П. Н. Нестеров}

Поступило

Ярославский государственный университет им. П. Г. Демидова

02.08.2005

E-mail: mathematix@mail.ru 